\title{
FDG-PET imaging to detect and characterize infectious disorders; an unavoidable path for the foreseeable future
}

\author{
Abass Alavi ${ }^{1}$ - Thomas J. Werner ${ }^{1}$ \\ Published online: 30 December 2016 \\ (C) Springer-Verlag Berlin Heidelberg 2016
}

In August 2016, we celebrated the 40th anniversary of the introduction of FDG-PET imaging and its enormous impact on the day-to-day practice of medicine [1]. The concept of imaging with FDG was primarily based upon wellestablished and scientifically proven high cerebral glycolytic activity by various methodologies. As such, early applications of FDG mostly dealt with assessment of neuropsychiatric disorders, which proved to be more sensitive and specific than conventional imaging techniques such as $\mathrm{CT}$ and MRI [2]. Soon thereafter, based on observations made by Warburg in the 1930's who demonstrated increased glycolysis in cancer cells, a few investigators expanded the use of FDG to diagnose and assess brain tumors [3]. These early research studies demonstrated the importance of FDG-PET in detecting brain cancer and characterizing its biological behavior. With the introduction of whole body PET instruments in the 1980's, efforts were made to investigate many other malignancies, which led to revolutionizing the fields of medical, radiation, and surgical oncology [4-6]. By adopting this technique to image the entire body, it was noted that tissues with inflammatory cells were also readily visualized by this methodology [7-9]. This observation eventually led to exploring the role of FDG-PET imaging in managing patients with infection and non-infectious inflammatory disorders. By the late 1990's and early 2000 's, PET was accepted as a reliable tool for managing

This Editorial Commentary refers to the article http://dx.doi. org/10.1007/s00259-016-3564-5

Abass Alavi

abass.alavi@uphs.upenn.edu

1 Department of Radiology, Hospital of the University of Pennsylvania, 3400 Spruce Street, Philadelphia, PA 19104, USA brain disorders and many malignancies, but there has been great resistance to determine the critical role of FDG-PET in inflammatory disorders. This has been a rather serious oversight by the medical community, particularly in the United States, where a disproportionately large number of FDGPET studies are performed compared to the rest of the world. This is quite unfortunate since therapeutic interventions for most malignant and central nervous system disorders are palliative in nature, while infectious and inflammatory disorders are treated and cured with modern treatment modalities. Therefore, there is a dire need to introduce and routinely adopt this powerful technology in patients with infection and inflammation in various phases of these disorders.

By now, it is proven that activated inflammatory cells at the site of infection have enhanced glycolysis compared to their inactive states and, therefore, utilize glucose and its analogues such as deoxyglucose as their main source of energy [10]. Many research studies over the past two decades have demonstrated that the degree of uptake of FDG by inflammatory cells is substantial in in-vitro settings, in animal experiments, and in human studies [11,12]. Therefore, this modality allows detection of infection with high sensitivity compared to other existing methods in medicine. Non-specific behavior of FDG leads to false-positive results when diagnosis of cancer is the main purpose in certain clinical scenarios. Some experimental data have demonstrated differences between malignant and inflammatory cells with regard to the dynamics of FDG uptake, which may allow improving the specificity of the results generated in patients with lesions of unknown underlying pathology $[11,12]$. While uptake of FDG in cancer tissues continues to rise for up to $4 \mathrm{~h}$, that of inflammatory cells plateaus after 60-90 min and may even decrease thereafter [13]. This is not a consistent finding and certain acute inflammatory reactions to bacteria may mimic malignant cells and reveal increasing activity over a few hours. 
Over the past two decades, the number of publications describing the results from both prospective and retrospective research studies has increased substantially, and as expected, there is clear evidence that FDG-PET imaging should become the primary method for detection of infection in different organs and various settings. Although most reports described in the literature deal with detection of infection in the lungs $[14,15]$ and the skeletal system [16-18], FDG-PET has been shown to be just as successful in other locations throughout the body [14]. The data published in the literature are very convincing and clearly demonstrate the critical role that this tracer can play in effective management of many common infectious disorders.

Over the past several decades, medicine has made substantial progress in treating infectious diseases, which is unparalleled in its efficacy compared with other domains in medicine. Particularly, the introduction of multiple antibiotics in the latter half of the 20th century has been revolutionary in minimizing or even eliminating morbidity and mortality related to many common bacterial infections. Unfortunately, the existing imaging techniques, including those provided by structural imaging modalities such as CT or MRI, lack both sensitivity and specificity in detecting and characterizing suspected infectious sites. In particular, these methodologies are quite unable to assess the degree of response to treatment and, therefore, have failed to personalize therapeutic interventions in serious and common infections such as tuberculosis $[19,20]$. In the era where personalized medicine is being heavily emphasized for effective management of serious maladies including infection, this is a major shortcoming and continues to result in over- and under-treating many infections. Therefore, FDG-PET, as a sensitive and specific imaging tool for assessing disease activity, can overcome these deficiencies and substantially improve patient outcome from these treatable diseases in the future [21]. This also will result in significant cost-saving and minimizing the financial burden of modern healthcare throughout the world.

Efforts have been made to image bacteria directly as an ideal approach for specific diagnosis of infection. Unfortunately, these efforts have failed and it is unlikely that, even with significant advances made in PET instrumentation, such a desirable possibility will be realized in the future for a few reasons. The spatial resolution of PET is in the range of several millimeters in phantom experiments and is substantially degraded in humans. This prevents detecting lesions that are smaller than 7-8 $\mathrm{mm}$ in size. Therefore, in order to detect bacterial infection, a significant number of these organisms should be amassed into a volume in the range of approximately one cubic centimeter. This is an unlikely possibility for most bacterial infections and can, therefore, explain the many failed attempts to visualize bacterial infections directly by this imaging approach [22]. In contrast, inflammatory reaction due to bacterial infection results in accumulation of a significant number of cells around the infected sites and can be visualized by current PET imaging machines. As such, the sensitivity of imaging infection/inflammation with FDG-PET is quite high, where more than $90 \%$ of the affected sites can be seen by this technique.

As noted above, one of the attributes of PET imaging is its ability to quantify disease activity with great precision and accuracy [23]. This capability allows physicians to monitor patients with infections during various phases of the disease. Based on the gained experience over the years, we have realized that sampling of a limited segment of the disease sites may underestimate the extent of the disease burden. In particular, when the disease is somewhat inhomogeneous in nature and behaves differently in various organs, quantitative techniques using a limited sample may underestimate the true information attainable from this powerful modality. Therefore, assessment of global disease activity may further enhance the role of PET during the course of the disease and, as such, allow effective use of this imaging technique. In particular, assessment of drug response will be best achieved by measuring overall disease activity in patients with a variety of infections, and this in turn will result in individualizing treatment in major infections such as tuberculosis, where drug resistance has posed a major challenge to infectious disease experts.

Increasingly, the role of conventional nuclear medicine techniques including radiolabeled white blood cell imaging is becoming limited in light of the advances being made with FDG-PET imaging for assessing infections. The process of labeling and administering white blood cells is quite cumbersome since it requires withdrawing a large volume of blood from the patient, isolating and labeling white cells in-vitro, and then administering them to the patient. Imaging is performed $24 \mathrm{~h}$ following administration of the labeled cells. The entire process is timeconsuming and very expensive. Additionally, there is a possibility of contamination of the administered cells by potent microorganisms as has been reported in the past. Furthermore, the dose of radiolabeled cells administered to the patients is typically below $1 \mathrm{mCi}$, resulting in images of poor quality that lack statistical validity, and, therefore, suboptimal sensitivity for detecting infection. Also, these images are planar in nature and cannot be used in conjunction with co-registered CT images as is the case with PET imaging. Furthermore, the radiation dose from Indium-111 labeled white blood cells to some organs is substantial (10 rads to the spleen) which is of concern since it is one of the highest in the practice of medical imaging. Finally, completing the study over 2 days is a major burden for these disabled patients compared to a single visit with FDG-PET imaging that can be completed in only a few hours. 
Therefore, the role of radiolabeled white blood cell imaging is rapidly diminishing in the day-to-day practice of medicine. This is particularly applicable to detection of infection following hip or knee arthroplasty where the results from white blood cell imaging are shown to be inferior to those from FDG-PET as documented by many studies published in the literature $[24,25]$.

Efforts are being made to label inflammatory cells by other tracers that are more specific and can be of value in certain settings. This approach may overcome some of the deficiencies that are associated with FDG-PET. It is know that FDG is picked up by certain organs such as the brain and heart. Therefore, it may not be feasible to reach optimal contrast between the inflammatory sites and the surrounding background activity. This is particularly true when infective endocarditis is present in patients with prosthetic valve infection $[26,27]$. Among potential agents for this purpose, radiolabeled nanoparticles appear to have a potential role for detection of inflammation in cardiac and possibly brain infections [28].

The article by Wareham et al. clearly demonstrates the critical role of FDG-PET imaging in assessing patients with suspected infection or recurrent cancer who have undergone organ transplants by accurately detecting and characterizing the infected sites. The data from this study clearly illustrates the impact that FDG-PET imaging can have in this setting and, therefore, justifies its routine employment in managing future patients with suspected infection following organ transplants. This will substantially improve the overall patient care and outcome and decrease the overall costs in both outand in-patient settings.

It is very desirable and timely for the community to adopt FDG-PET imaging for managing patients with a variety of infective disorders in the future. Without any equivocation, this will further enhance the role of PET imaging in medicine and demonstrate its unparalleled performance in this very common and potentially curable disorder. There has been resistance by the private and government insurance agencies to allow routine use of this very effective approach in various countries around the world. This primarily stems from the fact that very few prospective studies, which have enrolled a large number of patients, have been carried out to show the efficacy of FDG-PET and its cost-saving impact on the society. Our hope is that publications similar to that of Wareham et al. will encourage other centers to make an attempt to supplement the data presented by this group and convince the community that this is an unavoidable path for the foreseeable future. Until and unless these efforts are initiated, many patients with serious infectious disorders will suffer or even succumb to their disease due to suboptimal results provided by the existing imaging techniques.

\section{References}

1. Alavi A, Reivich M. Guest editorial: the conception of FDG-PET imaging. Semin Nucl Med. 2002;32:2-5. doi:10.1053/snuc.2002.29269.

2. Alavi A, Hirsch LJ. Studies of central nervous system disorders with single photon emission computed tomography and positron emission tomography: evolution over the past 2 decades. Semin Nucl Med. 1991;21:58-81.

3. Di Chiro G, DeLaPaz RL, Brooks RA, Sokoloff L, Kornblith PL, Smith $\mathrm{BH}$, et al. Glucose utilization of cerebral gliomas measured by $[18 \mathrm{~F}]$ fluorodeoxyglucose and positron emission tomography. Neurology. 1982;32:1323-9.

4. Wahl RL, Quint LE, Greenough RL, Meyer CR, White RI, Orringer MB. Staging of mediastinal non-small cell lung cancer with FDG PET, CT, and fusion images: preliminary prospective evaluation. Radiology. 1994;191:371-7. doi:10.1148/radiology.191.2.8153308.

5. Hustinx R, Benard F, Alavi A. Whole-body FDG-PET imaging in the management of patients with cancer. Semin Nucl Med. 2002;32:35-46. doi:10.1053/snuc.2002.29272.

6. Shankar LK, Hoffman JM, Bacharach S, Graham MM, Karp J, Lammertsma AA, et al. Consensus recommendations for the use of 18F-FDG PET as an indicator of therapeutic response in patients in national cancer institute trials. J Nucl Med. 2006;47:1059-66.

7. Alavi A, Buchpiguel CA, Loessner A. Is there a role for FDG PET imaging in the management of patients with sarcoidosis? J Nucl Med. 1994;35:1650-2.

8. Lewis PJ, Salama A. Uptake of fluorine-18-fluorodeoxyglucose in sarcoidosis. J Nucl Med. 1994;35:1647-9.

9. Larson SM. Cancer or inflammation? a holy grail for nuclear medicine. J Nucl Med. 1994;35:1653-5.

10. Maratou E, Dimitriadis G, Kollias A, Boutati E, Lambadiari V, Mitrou P, et al. Glucose transporter expression on the plasma membrane of resting and activated white blood cells. Eur J Clin Investig. 2007;37:282-90. doi:10.1111/j.1365-2362.2007.01786.x.

11. Zhuang H, Pourdehnad M, Lambright ES, Yamamoto AJ, Lanuti $\mathrm{M}$, Li P, et al. Dual time point 18F-FDG PET imaging for differentiating malignant from inflammatory processes. J Nucl Med. 2001;42:1412-7.

12. Zhuang H, Alavi A. 18-fluorodeoxyglucose positron emission tomographic imaging in the detection and monitoring of infection and inflammation. Semin Nucl Med. 2002;32: 47-59. doi:10.1053/snuc.2002.29278.

13. Basu S, Kung J, Houseni M, Zhuang H, Tidmarsh GF, Alavi A. Temporal profile of fluorodeoxyglucose uptake in malignant lesions and normal organs over extended time periods in patients with lung carcinoma: implications for its utilization in assessing malignant lesions. Q J Nucl Med Mol Imaging. 2009;53:9-19.

14. Chacko TK, Zhuang H, Nakhoda KZ, Moussavian B, Alavi A. Applications of fluorodeoxyglucose positron emission tomography in the diagnosis of infection. Nucl Med Commun. 2003;24:615-24. doi:10.1097/01.mnm.0000075189.60210.df.

15. Vorster M, Sathekge MM, Bomanji J. Advances in imaging of tuberculosis: the role of (1)(8)F-FDG PET and PET/CT. Curr Opin Pulm Med. 2014;20:287-93. doi:10.1097/MCP.0000000000000043.

16. Ichiya Y, Kuwabara Y, Sasaki M, Yoshida T, Akashi Y, Murayama $\mathrm{S}$, et al. FDG-PET in infectious lesions: the detection and assessment of lesion activity. Ann Nucl Med. 1996;10:185-91.

17. Meller J, Sahlmann CO, Liersch T, Hao Tang P, Alavi A. Nonprosthesis orthopedic applications of (18)F fluoro-2-deoxyD-glucose PET in the detection of osteomyelitis. Radiol Clin N Am. 2007;45:719-33. doi:10.1016/j.rcl.2007.05.011. vii-viii.

18. Kwee TC, Kwee RM, Alavi A. FDG-PET for diagnosing prosthetic joint infection: systematic review and metaanalysis. Eur J Nucl Med Mol Imaging. 2008;35:2122-32. doi:10.1007/s00259-008-0887-x. 
19. Martinez V, Castilla-Lievre MA, Guillet-Caruba C, Grenier G, Fior R, Desarnaud S, et al. (18)F-FDG PET/CT in tuberculosis: an early non-invasive marker of therapeutic response. Int J Tuberc Lung Dis. 2012;16:1180-5. doi:10.5588/ijtld.12.0010.

20. Chen RY, Dodd LE, Lee M, Paripati P, Hammoud DA, Mountz JM, et al. PET/CT imaging correlates with treatment outcome in patients with multidrug-resistant tuberculosis. Sci Transl Med. 2014. doi:10.1126/scitranslmed.3009501.

21. Glaudemans AW, de Vries EF, Galli F, Dierckx RA, Slart RH, Signore A. The use of (18)F-FDG-PET/CT for diagnosis and treatment monitoring of inflammatory and infectious diseases. Clin Dev Immunol. 2013;2013:623036. doi:10.1155/2013/623036.

22. Zhang XM, Zhang HH, McLeroth P, Berkowitz RD, Mont MA, Stabin MG, et al. [(124)I]FIAU: Human dosimetry and infection imaging in patients with suspected prosthetic joint infection. Nucl Med Biol. 2016;43:273-9. doi:10.1016/j.nucmedbio.2016.01.004.

23. Houshmand S, Salavati A, Hess S, Werner TJ, Alavi A, Zaidi H. An update on novel quantitative techniques in the context of evolving whole-body PET imaging. PET Clin. 2015;10:45-58. doi:10.1016/j.cpet.2014.09.004.

24. Basu S, Kwee TC, Saboury B, Garino JP, Nelson CL, Zhuang H, et al. FDG PET for diagnosing infection in hip and knee prostheses: prospective study in 221 prostheses and subgroup comparison with combined (111)In-labeled leukocyte/(99m)Tc-sulfur colloid bone marrow imaging in 88 prostheses. Clin Nucl Med. 2014;39:60915. doi:10.1097/RLU.0000000000000464.

25. Kwee TC, Basu S, Alavi A. Letter: opinion on favoring labeled leukocyte imaging over 18F-FDG-PET for diagnosing prosthetic joint infection: rectifying ongoing misconceptions. J Nucl Med. 2016. doi:10.2967/jnumed.116.181461.

26. Millar BC, Prendergast BD, Alavi A, Moore JE. 18FDG-positron emission tomography (PET) has a role to play in the diagnosis and therapy of infective endocarditis and cardiac device infection. Int J Cardiol. 2013;167:1724-36. doi:10.1016/j.ijcard.2012.12.005.

27. Habib G, Lancellotti P, Antunes MJ, Bongiorni MG, Casalta JP, Del Zotti F, et al. 2015 ESC guidelines for the management of infective endocarditis: the task force for the management of infective endocarditis of the European society of cardiology (ESC). endorsed by: European association for cardio-thoracic surgery (EACTS), the European association of nuclear medicine (EANM). Eur Heart J. 2015;36:3075-128. doi:10.1093/eurheartj/ehv319.

28. de Barros AB, Tsourkas A, Saboury B, Cardoso VN, Alavi A. Emerging role of radiolabeled nanoparticles as an effective diagnostic technique. EJNMMI Res. 2012;2:39. doi:10.1186/2191-219 $\mathrm{X}-2-39$. 